\title{
Análisis taxonómico de las especies mexicanas de Belonuchus Nordmann del grupo ephippiatus (Coleoptera: Staphylinidae)
}

\section{Taxonomic analysis of the Mexican species of Belonuchus Nordmann of the ephippiatus group (Coleoptera: Staphylinidae)}

\author{
Juan Márquez ${ }^{1, *}$, Julieta Asiain ${ }^{1} \&$ José Luis Navarrete-Heredia ${ }^{2}$ \\ ${ }^{1}$ Laboratorio de Sistemática Animal, Centro de Investigaciones Biológicas, UAEH, Km 4.5, carretera Pachuca-Tulancingo s/n, \\ Ciudad del Conocimiento, Col. Carboneras, CP 42184, Mineral de la Reforma, Hidalgo, México. \\ ${ }^{2}$ Entomología, Centro de Estudios en Zoología, CUCBA, Universidad de Guadalajara, Apdo. Postal 134, 45100, Zapopan, \\ Jalisco, México. \\ ${ }^{*}$ E-mail: marquezorum@gmail.com
}

\begin{abstract}
RESUMEN
Se analizaron 117 ejemplares procedentes de ocho estados y 22 localidades de México cuya identificación taxonómica era dudosa para asignarlos a Belonuchus ephippiatus o B. erythropterus. La finalidad de este trabajo fue analizar los principales caracteres reportados en la literatura para separar a las dos especies mencionadas y decidir si se trata de una o dos entidades. El análisis incluyó la medición de diferentes partes del cuerpo y las proporciones entre ellas, pero éste se focalizó en la longitud total del cuerpo, el número de puntuaciones que forman cada una de las dos hileras centrales del pronoto, la longitud del edéago y su proporción con relación a la longitud del cuerpo, ya que son los principales caracteres indicados en la literatura que permiten separar a las dos especies. Los resultados muestran que no es posible distinguir dos especies con ninguno de los caracteres analizados, combinados o solos, por lo que se propuso por segunda ocasión en la historia de estas especies, que $B$. erythropterus es sinónimo junior de B. ephippiatus. Se discuten aspectos sobre la enorme variación morfológica observada en $B$. ephippiatus, su distribución geográfica y su biología. Además, se describe una especie nueva del mismo grupo de especies y se distingue de B. ephippiatus con base en caracteres morfológicos. Finalmente, se aportan esquemas y fotografías que ayudan a su reconocimiento.
\end{abstract}

Palabras clave: Belonuchus moroni sp. nov., México, sinonimia, taxonomía.

\begin{abstract}
We analyzed 117 specimens from eight Mexican states and 22 localities whose taxonomic identification was doubtful to assign them to Belonuchus ephippiatus or B. erythropterus. The goal of this paper is to analyze the most important characters reported in the literature to separate both species and decide if it is one or two entities. The analysis included the measurement of different parts of the body and the proportions between them, however, it was focused on the total body length, the number of punctures that form each of the two central rows of the pronotum, the aedeagus length and its proportion in relation to the body length, because they are the most important characters indicated in the literature that allow to separate the both species. The results showed that is not possible to distinguish the two species with any of the characters analyzed, combined or alone, so we proposed for the second time in the history of these species, that $B$. erythropterus is a junior synonym of B. ephippiatus. Aspects of the great morphological variation observed in B. ephippiatus, its geographic distribution and its biology are discussed. Moreover, a new species of the same species group was described and it was distinguished from $B$. ephippiatus based on morphological characters. Finally, we are providing schemes and photographs that help its recognition.
\end{abstract}

KeYwords: Belonuchus moroni sp. nov., Mexico, synonymy, taxonomy.

\section{INTRODUCCIÓN}

En México se conocen 27 especies de Belonuchus y se tiene conocimiento de la existencia de algunas especies nuevas que aún no se han descrito formalmente porque es necesario realizar un análisis taxonómico integral de todas ellas (Navarrete-Heredia et al. 2002; Rodríguez \& Navarrete-Heredia 2016). En América, Colombia ocupa el primer lugar en número de especies con 35, México el segundo y Brasil el tercero (25 especies), aunque son países que también comparten la falta de estudios sistemáticos que ayuden a la identificación de sus especies (Herman 
2001). En contraste, en Guatemala se han registrado solo 7 especies, cifra que podría aumentar considerablemente en estudios taxonómicos futuros (Herman 2001).

Las especies Mexicanas de Belonuchus se han estudiado principalmente en análisis faunísticos, donde ha sido frecuente el uso de trampas cebadas con carroña, y con menor intensidad en trabajos taxonómicos (RuízLizárraga 1993, Navarrete-Heredia 1995, 1996, JiménezSánchez 1998, Santiago-Jiménez 1999, Márquez 2001, 2003, 2004, 2006, Márquez et al. 2004, Ramírez-Reséndiz 2009, Huerta-Camacho 2010, Pedraza et al. 2010, Asiain et al. 2011, Márquez \& Asiain 2012, entre otros). Los resultados de estos estudios han revelado que constituyen un grupo depredador importante porque algunas especies son abundantes en sustratos en descomposición (carroña, pulpa de café, nopales y magueyes en descomposición, etc.) y pueden consumir larvas de otros grupos de insectos, principalmente dípteros.

Algunas de las especies conocidas de México que se comparten con el sur de E.U.A. fueron incluidas en el estudio de las especies de Philonthina realizado por Smetana (1995), quien además redescribió el género Belonuchus y propuso el arreglo de las especies en grupos. Uno de estos grupos es ephippiatus, el cual se caracteriza por la cabeza carente de carena en la zona temporal, hileras centrales en el pronoto con 5 ó 6 a 8 puntuaciones cada una, tarsómeros medios y posteriores con excavación media longitudinal en su superficie dorsal, área elevada entre las dos líneas basales de los terguitos abdominales visibles 2 y 3 con microescultura en forma de submalla, y los parámeros del edéago reducidos a una placa corta semi-fusionada con el lóbulo medio (Smetana 1995).

Este grupo incluye dos especies, B. ephippiatus Say, 1820 y B. erythropterus Solsky, 1868, las cuales se han registrado con cierta frecuencia en diversas localidades de México (Navarrete-Heredia et al. 2002, Márquez 2006). Sin embargo, la distinción taxonómica entre ellas con base en los caracteres proporcionados por Smetana (1995) es difícil y nos ha motivado a reanalizar si se trata realmente de dos especies muy similares entre sí o se trata de una sola especie morfológicamente variable.

Por lo anterior, en este trabajo se analiza una serie de ejemplares procedentes de varios estados y localidades para discernir si se trata de una o de dos especies y proponer los cambios taxonómicos pertinentes. Además, se describe una especie nueva con base en caracteres morfológicos y morfométricos, sin que hasta el momento se cuente con ninguna otra especie conocida o por describir del grupo ephippiatus.

\section{MATERIALES Y MÉTODOS}

Los ejemplares estudiados de las dos especies conocidas se encuentran depositados en la Colección de Coleoptera de la
Universidad Autónoma del Estado de Hidalgo (CC-UAEH, Mineral de la Reforma, Hidalgo, México) y provienen de los estados de Aguascalientes, Baja California, Estado de México, Hidalgo, Michoacán, Morelos, Puebla y Querétaro. La mayoría de los ejemplares estaban previamente identificados como B. ephippiatus, pero los de mayor longitud estaban como $B$. erythropterus. Con la finalidad de realizar el análisis para discernir si se trata de una o dos especies y las diferencias entre éstas, no se asignó ninguno de los dos nombres a los especímenes estudiados y se procedió a la medición de sus estructuras diagnósticas con ayuda de un micrómetro colocado en el ocular del microscopio estereoscópico.

Algunas mediciones no resultaron relevantes en este análisis, por ejemplo, la longitud de las mandíbulas respecto a la cabeza o la proporción largo-ancho del pronoto, pues no había diferencias que ayudaran a resolver el problema de si se trata de una o dos especies; sin embargo, sí son parte de la variación. Por lo anterior, el análisis se dirigió a las estructuras que de acuerdo con Smetana (1995) son relevantes para distinguir B. ephippiatus de B. erythropterus, básicamente la longitud total del cuerpo, que fue medida trazando una línea media imaginaria desde el borde anterior de la cabeza, excluyendo las mandíbulas, hasta el borde posterior del último segmento abdominal visible, excluyendo los estilos, y la longitud del edéago, que fue medida trazando una línea media imaginaria desde el borde anterior hasta el posterior del lóbulo medio. Además se contó el número de puntuaciones en cada hilera central del pronoto, considerando en todos los casos sólo una puntuación cercana al borde anterior, en donde se puede confundir con las puntuaciones cercanas a ese borde, en adición a las que se encuentran claramente separadas de éste.

Los resultados se presentan en cuatro partes. La primera corresponde a los datos de longitud total y número de puntuaciones en las dos hileras centrales del pronoto de 59 machos. Además, en 50 de ellos se pudo medir la longitud del edéago. Se establecieron tres categorías del tamaño de los ejemplares: chicos, medianos y grandes, con base en la diferencia que se presentó entre el ejemplar de menor longitud total del cuerpo respecto al de mayor longitud. Esta diferencia se dividió en tres partes iguales que permitió tener un rango de medidas pequeñas para los ejemplares chicos, otro de medidas intermedias para los ejemplares medianos y el tercero de medidas mayores para los ejemplares grandes.

Para analizar qué tan grande o chico es cada edéago, se consideró que su longitud total por sí sola no necesariamente lo determina, sino que se requiere conocer qué proporción representa o equivale ese edéago respecto al tamaño del organismo completo. Hipotéticamente hablando, podemos comparar un primer organismo cuya longitud total sea de $100 \mathrm{~mm}$ y que su edéago sea de $10 \mathrm{~mm}$ de longitud, lo que en proporción el edéago mide el $10 \%$ de la longitud del ejemplar; mientras que un segundo ejemplar de menor 
longitud, por ejemplo de $80 \mathrm{~mm}$, y un edéago de $9 \mathrm{~mm}$ de longitud, representaría el 11,25\% de la longitud del ejemplar. En este caso hipotético, es correcto que el primer ejemplar tiene un edéago más grande que el segundo, pero si se analiza desde el punto de vista de sus proporciones, el segundo ejemplar tiene un edéago más grande que el primero. Nuestras observaciones previas de los ejemplares estudiados sugieren que podría ocurrir lo explicado en el caso hipotético, por lo cual también se analizan las proporciones (en porcentaje) entre la longitud del edéago y la del organismo al que pertenece. Siguiendo el mismo criterio explicado para determinar cuáles ejemplares se consideraron de tamaño chico, mediano y grande, se establecieron también estas tres categorías para el tamaño de los edéagos, pero desde el punto de vista de su proporción respecto al tamaño del ejemplar al que pertenece.

La segunda parte de resultados corresponde al análisis de 58 hembras, en el cual se incluyó el número de puntuaciones en cada hilera central del pronoto y la longitud total del cuerpo. También se establecieron las tres categorías de tamaño de los ejemplares (chica, mediana y grande) siguiendo el mismo criterio aplicado en los machos. En la tercera parte se presentan los datos de machos y hembras de cada una de las tres localidades mejor representadas en la muestra, las cuales pertenecen a los estados de Querétaro (Peña de Bernal: 25 machos y 21 hembras) e Hidalgo (Barranca de Metztitlán: 9 machos y 4 hembras; Sierra de Pitos: 8 machos y 2 hembras). La finalidad de este tercer análisis fue verificar si en áreas geográficas particulares se presenta o no el mismo patrón que al considerar todos los datos.

En la cuarta parte de resultados se presenta la información taxonómica, que incluye la propuesta de sinonimia, la variación morfológica observada en B. ephippiatus diferente a la mencionada en las partes previas de resultados, y la descripción de una especie nueva del grupo ephippiatus, que incluye una diagnosis con información suficiente para distinguir las dos especies que integrarán dicho grupo. La descripción de la nueva especie se basó en seis ejemplares donados por E. Pfeiler. Sus datos de colecta se encuentran entre comillas en la parte de material tipo, donde también se indican las colecciones de depósito de los tipos, que además de la CC-UAEH, incluye a la Colección Entomológica, del Centro de Estudios en Zoología, CUCBA, Universidad de Guadalajara, Zapopan, Jalisco, México (CZUG).

\section{RESULTADOS}

\section{ANÁLISIS DE LA VARIACIÓN EN MACHOS}

A partir de los datos de la Tabla 1 se obtuvieron los resultados de los caracteres de los machos, los cuales se sintetizan en la Tabla 2. La diferencia en longitud total entre el ejemplar más pequeño y el más grande fue de $7,9 \mathrm{~mm}$. La mayor proporción de machos fue de tamaño mediano $(52,5 \%)$, seguida por el tamaño grande $(24,5 \%)$ y el tamaño chico presentó la menor proporción (22 \%) (Fig. 1a-c). El 18,6 $\%$ de los ejemplares presentó más de 5 puntuaciones en las hileras centrales del pronoto. Con relación a la proporción que representa el edéago respecto al cuerpo de los organismos, en la mayoría ésta fue mediana ( $52 \%$ ), seguida por la proporción chica $(36 \%)$ y la proporción grande fue la menos común (12\%). Los únicos 6 ejemplares que presentaron edéago grande en proporción al cuerpo son de tamaño chico (2) y mediano (4). De todos los machos, sólo 11 tuvieron más de 5 puntuaciones en cada hilera central del pronoto, 7 de ellos son de tamaño grande y 4 de tamaño mediano, y sólo uno de los 11 mostró un edéago grande respecto al cuerpo, en el resto éste es mediano o chico.

ANÁLISIS DE LA VARIACIÓN EN HEMBRAS

A partir de los datos de la Tabla 3 se obtuvieron los resultados de los caracteres de las hembras, éstos se sintetizan en la Tabla 2. La diferencia entre la longitud total de la hembra más chica y la más grande fue de $11,0 \mathrm{~mm}$. La mayor cantidad de hembras fueron de tamaño chico $(62,1 \%)$, en segundo lugar fueron de tamaño mediano $(27,6 \%)$ y en tercer lugar de tamaño grande (10,3\%) (Fig. 1d-f). En 10 hembras $(17,2 \%)$ se presentaron más de 5 puntuaciones en cada hilera central del pronoto, 5 de ellas fueron de tamaño grande, 3 de tamaño mediano y 2 de tamaño chico.

ANÁLISIS DE LA VARIACIÓN EN MACHOS Y HEMBRAS DE LAS TRES LOCALIDADES MEJOR REPRESENTADAS

Los ejemplares analizados provienen de 8 estados del país de un total de 13 donde ambas especies han sido registradas (Tablas 1, 3 y 4, Fig. 2). En la Tabla 4 se sintetizan los datos obtenidos de la variación morfológica de machos y hembras provenientes de las tres localidades mejor representadas en la muestra general. La localidad "La Peña de Bernal", municipio de Bernal, estado de Querétaro, es la mejor representada en la muestra con 46 ejemplares que equivalen al 39,3\% del total de organismos (117). De ellos, 25 son machos (42,3\% del total de machos) y 21 son hembras (36,2\% del total de hembras).

La segunda localidad mejor representada es la Reserva de la Biósfera Barranca de Metztitlán, municipio de Metztitlán, estado de Hidalgo, con 13 organismos que equivalen a $11,11 \%$ del total. Nueve son machos $(15,25 \%$ del total de machos) y 4 son hembras (6,9\% del total de hembras).

La tercera localidad mejor representada es la Sierra de Pitos, municipio de Zempoala, estado de Hidalgo, con 10 ejemplares que equivalen a $8,54 \%$ del total, 8 machos $(13,56$ $\%$ de los machos) y 2 hembras (3,45\% de las hembras). El resto de las localidades están poco representadas, excepto por una del estado de Morelos, pero son todas hembras (10). 
TAXONOMÍA

Propuesta de sinonimia. Con base en el análisis de los caracteres estudiados proponemos que el nombre $B$. erythropterus es sinónimo junior de $B$. ephippiatus. En la discusión se proporciona mayor información que sustenta esta propuesta.

Variación. Otras medidas que forman parte de la variación morfológica de $B$. ephippiatus, son: longitud de las mandíbulas respecto a la longitud de la cabeza: machos, rango $0,77-1,02$, promedio 0,90 ; hembras, rango 0,88-1,06, promedio 0,91; proporción largo-ancho de la cabeza: machos, rango $0,82-0,94$, promedio 0,88 ; hembras, rango $0,81-0,93$, promedio 0,86 ; proporción largo-ancho del pronoto: machos, rango $0,95-1,16$, promedio 1,04 ; hembras, rango $0,9-1,16$, promedio 1,08 . No se apreció relación de las medidas anteriores con el sexo.

Belonuchus moroni sp. nov. (Fig. 3a)

Holotipo macho (CC-UAEH): "México: Baja California Norte, municipio de Ensenada, La Sepultura, cerca de N $29^{\circ} \mathrm{W} 114^{\circ}$, en cactus senita (Lophocereus schottii), julio de 2003, E. Pfeiler col.” El holotipo carece de los antenómeros 7 a 11 de la antena derecha, la cabeza está un poco dañada del lado posterior izquierdo y carece de la pata media derecha. Paratipos (cinco hembras): mismos datos que el holotipo (2, CC-UAEH; una hembra sin cabeza y con el pronoto dañado). "México: Baja California Sur, municipio de Loreto, Armenta, carca de N 26․, W $111^{\circ}$, en cactus senita (Lophocereus schottii), julio de 2003, E. Pfeiler col." (2, CC-UAEH; 1, CZUG).

TABLa 1. Medidas de longitud total del cuerpo (LT), longitud del edéago (LE) y número de puntuaciones en cada hilera central del pronoto de 59 machos de Belonuchus ephippiathus y/o B. erythropterus. Entre paréntesis se anota si se trata de ejemplares chicos $(\mathrm{CH})$, medianos (ME) o grandes (GR) en longitud total y en la proporción LE / LT. / Measurements of total body length (LT), aedeagus length (LE) and number of punctures in each central row of the pronotum of 59 males of Belonuchus ephippiathus and/or B. erythropterus. In parentheses it is written whether the specimens are small (CH), medium (ME) or large (GR) in total length and in the LE / LT ratio.

\begin{tabular}{|c|c|c|c|c|}
\hline Estado / Municipio (localidad) & $\begin{array}{l}\text { Longitud total } \\
\text { del cuerpo } \\
\text { (LT) (mm) }\end{array}$ & $\begin{array}{l}\text { Longitud del } \\
\text { edéago (LE) (mm) }\end{array}$ & $\begin{array}{l}\text { Proporción LE / } \\
\text { LT }(\%)\end{array}$ & $\begin{array}{c}\text { Número de } \\
\text { puntuaciones } \\
\text { en cada hilera } \\
\text { central del pronoto } \\
\text { diferente a 5:5 }\end{array}$ \\
\hline 1. Baja California / Ensenada (Cataviña) & $12,2(\mathrm{CH})$ & 1,34 & $11,0(\mathrm{ME})$ & \\
\hline $\begin{array}{l}\text { 2. Estado de México / Otumba (carretera a Tuxpan, } \\
\text { desviación a Otumba) }\end{array}$ & $13,7(\mathrm{ME})$ & 1,32 & 11,7 (ME) & \\
\hline $\begin{array}{l}\text { 3. Estado de México / Otumba (carretera a Tuxpan, } \\
\text { desviación a Otumba) }\end{array}$ & $14,9(\mathrm{ME})$ & 1,67 & $11,2(\mathrm{ME})$ & \\
\hline 4. Hidalgo / Acatlán (Loma Larga) & $14,4(\mathrm{ME})$ & 1,35 & $9,4(\mathrm{CH})$ & \\
\hline 5. Hidalgo / Ajacuba (Ajacuba) & $15,2(\mathrm{ME})$ & 1,55 & $10,2(\mathrm{ME})$ & $6: 6$ \\
\hline 6. Hidalgo / Epazoyucan (5 km SE) & $13,1(\mathrm{ME})$ & Sin edéago (s/e) & --- & \\
\hline $\begin{array}{l}\text { 7. Hidalgo / Metztitlán (reserva de la biósfera Barranca } \\
\text { de Metztitlán: RBBM) }\end{array}$ & $16,8(\mathrm{GR})$ & 1,62 & $9,7(\mathrm{CH})$ & $6: 7$ \\
\hline 8. Hidalgo / Metztitlán (RBBM) & 15,7 (GR) & 1,60 & $10,2(\mathrm{ME})$ & $6: 6$ \\
\hline 9. Hidalgo / Metztitlán (RBBM) & $14,6(\mathrm{ME})$ & 1,25 & $8,6(\mathrm{CH})$ & \\
\hline 10. Hidalgo / Metztitlán (RBBM) & $15,2(\mathrm{ME})$ & 1,45 & $9,5(\mathrm{CH})$ & \\
\hline 11. Hidalgo / Metztitlán (RBBM) & $13,0(\mathrm{ME})$ & 1,40 & $10,8(\mathrm{ME})$ & \\
\hline 12. Hidalgo / Metztitlán (RBBM) & $16,3(\mathrm{GR})$ & 1,60 & $9,8(\mathrm{CH})$ & $6: 6$ \\
\hline 13. Hidalgo / Metztitlán (RBBM) & $14,4(\mathrm{ME})$ & 1,45 & $10,1(\mathrm{CH})$ & \\
\hline 14. Hidalgo / Metztitlán (RBBM) & $14,6(\mathrm{ME})$ & 1,42 & $9,8(\mathrm{CH})$ & \\
\hline 15. Hidalgo / Metztitlán (RBBM) & $12,0(\mathrm{CH})$ & $\mathrm{s} / \mathrm{e}$ & --- & \\
\hline 16. Hidalgo / Tolcayucan (cerro Mambrú) & $16,8(\mathrm{GR})$ & $\mathrm{s} / \mathrm{e}$ & --- & \\
\hline 17. Hidalgo / Tolcayucan (cerro Mambrú) & $13,6(\mathrm{ME})$ & 1,67 & $12,3(\mathrm{GR})$ & \\
\hline 18. Hidalgo / Tolcayucan (cerro Mambrú) & $17,1(\mathrm{GR})$ & 1,79 & $10,5(\mathrm{ME})$ & \\
\hline 19. Hidalgo / Zempoala (Sierra de Pitos) & $10,3(\mathrm{CH})$ & 1,25 & $12,1(\mathrm{GR})$ & \\
\hline 20. Hidalgo / Zempoala (Sierra de Pitos) & $17,5(\mathrm{GR})$ & $\mathrm{s} / \mathrm{e}$ & --- & $5: 7$ \\
\hline 21. Hidalgo / Zempoala (Sierra de Pitos) & 16,4 (GR) & 1,70 & $10,4(\mathrm{ME})$ & \\
\hline
\end{tabular}




\begin{tabular}{|c|c|c|c|c|}
\hline Estado / Municipio (localidad) & $\begin{array}{l}\text { Longitud total } \\
\text { del cuerpo } \\
\text { (LT) (mm) }\end{array}$ & $\begin{array}{l}\text { Longitud del } \\
\text { edéago (LE) (mm) }\end{array}$ & $\begin{array}{l}\text { Proporción LE / } \\
\text { LT }(\%)\end{array}$ & $\begin{array}{c}\text { Número de } \\
\text { puntuaciones } \\
\text { en cada hilera } \\
\text { central del pronoto } \\
\text { diferente a 5:5 }\end{array}$ \\
\hline 22. Hidalgo / Zempoala (Sierra de Pitos) & $15,2(\mathrm{ME})$ & 1,77 & $11,7(\mathrm{ME})$ & $7: 6$ \\
\hline 23. Hidalgo / Zempoala (Sierra de Pitos) & $14,5(\mathrm{ME})$ & 1,50 & $10,3(\mathrm{ME})$ & \\
\hline 24. Hidalgo / Zempoala (Sierra de Pitos) & $16,3(\mathrm{GR})$ & 1,55 & $9,5(\mathrm{CH})$ & \\
\hline 25. Hidalgo / Zempoala (Sierra de Pitos) & 15,7 (GR) & 1,37 & $8,7(\mathrm{CH})$ & \\
\hline 26. Hidalgo / Zempoala (Sierra de Pitos) & $11,6(\mathrm{CH})$ & 1,32 & $11,4(\mathrm{ME})$ & \\
\hline 27. Hidalgo /Cuautepec de Hinojosa (El Campanario) & $13,6(\mathrm{ME})$ & 1,82 & $13,4(\mathrm{GR})$ & $8: 6$ \\
\hline 28. Hidalgo /Cuautepec de Hinojosa (El Campanario) & $17,4(\mathrm{GR})$ & 1,92 & $11,0(\mathrm{ME})$ & $6: 6$ \\
\hline 29. Michoacán / Tzintzuntzan (San Pedro Cucuchuchu) & $12,4(\mathrm{CH})$ & 1,29 & $10,4(\mathrm{ME})$ & \\
\hline $\begin{array}{l}\text { 30. Puebla / San Nicolás de Buenos Aires (carretera a } \\
\text { Xalapa, km 63) }\end{array}$ & $15,2(\mathrm{ME})$ & 1,62 & $10,7(\mathrm{ME})$ & \\
\hline $\begin{array}{l}\text { 31. Puebla / San Nicolás de Buenos Aires (carretera a } \\
\text { Xalapa, km 63) }\end{array}$ & $11,8(\mathrm{CH})$ & 1,57 & 13,3 (GR) & \\
\hline $\begin{array}{l}\text { 32. Puebla / San Nicolás de Buenos Aires (carretera a } \\
\text { Xalapa, km 63) }\end{array}$ & $13,0(\mathrm{ME})$ & $\mathrm{s} / \mathrm{e}$ & --- & \\
\hline $\begin{array}{l}\text { 33. Puebla / San Nicolás de Buenos Aires (carretera a } \\
\text { Xalapa, km 63) }\end{array}$ & $13,2(\mathrm{ME})$ & 1,62 & $12,3(\mathrm{GR})$ & \\
\hline 34. Puebla: Tehuacán (La Mesa) & 13,5 (ME) & 1,37 & 10,2 (ME) & \\
\hline 35. Querétaro / Bernal (Peña) & $16,2(\mathrm{GR})$ & 1,55 & $9,6(\mathrm{CH})$ & \\
\hline 36. Querétaro / Bernal (Peña) & $17,9(\mathrm{GR})$ & 1,95 & $10,9(\mathrm{ME})$ & $8: 9$ \\
\hline 37. Querétaro / Bernal (Peña) & $18,2(\mathrm{GR})$ & 1,86 & $10,2(\mathrm{ME})$ & $6: 6$ \\
\hline 38. Querétaro / Bernal (Peña) & $14,3(\mathrm{ME})$ & 1,45 & $10,1(\mathrm{CH})$ & \\
\hline 39. Querétaro / Bernal (Peña) & $16,6(\mathrm{GR})$ & 1,77 & 10,7 (ME) & \\
\hline 40. Querétaro / Bernal (Peña) & $13,4(\mathrm{ME})$ & 1,50 & $11,2(\mathrm{ME})$ & \\
\hline 41. Querétaro / Bernal (Peña) & $14,6(\mathrm{ME})$ & 1,47 & $10,1(\mathrm{CH})$ & \\
\hline 42. Querétaro / Bernal (Peña) & $17,1(\mathrm{GR})$ & 1,50 & $8,8(\mathrm{CH})$ & $6: 6$ \\
\hline 43. Querétaro / Bernal (Peña) & $14,0(\mathrm{ME})$ & 1,67 & $12,0(\mathrm{GR})$ & \\
\hline 44. Querétaro / Bernal (Peña) & $12,0(\mathrm{CH})$ & 1,30 & $10,8(\mathrm{ME})$ & \\
\hline 45. Querétaro / Bernal (Peña) & $11,1(\mathrm{CH})$ & 1,27 & $11,5(\mathrm{ME})$ & \\
\hline 46. Querétaro / Bernal (Peña) & $12,9(\mathrm{CH})$ & 1,25 & $9,7(\mathrm{CH})$ & \\
\hline 47. Querétaro / Bernal (Peña) & $12,6(\mathrm{CH})$ & 1,40 & $11,1(\mathrm{ME})$ & \\
\hline 48. Querétaro / Bernal (Peña) & $11,9(\mathrm{CH})$ & 1,37 & 11,6 (ME) & \\
\hline 49. Querétaro / Bernal (Peña) & $13,8(\mathrm{ME})$ & 1,35 & $9,8(\mathrm{CH})$ & \\
\hline 50. Querétaro / Bernal (Peña) & $13,5(\mathrm{ME})$ & 1,40 & $10,4(\mathrm{ME})$ & \\
\hline 51. Querétaro / Bernal (Peña) & $12,7(\mathrm{CH})$ & 1,32 & $10,4(\mathrm{ME})$ & \\
\hline 52. Querétaro / Bernal (Peña) & $14,3(\mathrm{ME})$ & 1,37 & $9,6(\mathrm{CH})$ & \\
\hline 53. Querétaro / Bernal (Peña) & $14,8(\mathrm{ME})$ & 1,52 & $10,3(\mathrm{ME})$ & \\
\hline 54. Querétaro / Bernal (Peña) & $15,5(\mathrm{ME})$ & 1,39 & $9,0(\mathrm{CH})$ & \\
\hline 55. Querétaro / Bernal (Peña) & $15,2(\mathrm{ME})$ & 1,40 & $9,2(\mathrm{CH})$ & \\
\hline 56. Querétaro / Bernal (Peña) & $13,6(\mathrm{ME})$ & $\mathrm{s} / \mathrm{e}$ & --- & \\
\hline 57. Querétaro / Bernal (Peña) & $11,8(\mathrm{CH})$ & $\mathrm{s} / \mathrm{e}$ & --- & \\
\hline 58. Querétaro / Bernal (Peña) & 13,2 (ME) & $\mathrm{s} / \mathrm{e}$ & --- & \\
\hline 59. Querétaro / Bernal (Peña) & $14,4(\mathrm{ME})$ & $\mathrm{s} / \mathrm{e}$ & --- & \\
\hline
\end{tabular}


Taxonomía de Belonuchus grupo ephippiatus: MárquEZ, J. ET AL.

TABLA 2. Análisis de la variación morfológica de los machos y hembras de Belonuchus ephippiathus y/o B. erythropterus. / Analysis of the morphological variation of males and females of Belonuchus ephippiathus and/or B. erythropterus.

\begin{tabular}{|c|c|}
\hline PARÁmetros EN MACHOS & VALORES OBTENIDOS \\
\hline Longitud total del cuerpo $(n=59)$ & $\begin{array}{l}\text { Promedio: } 14,35 \mathrm{~mm} \\
\text { Rango: } 10,3-18,2 \mathrm{~mm} \\
\text { En rangos de } 1 \mathrm{~mm} \text {, los } 2 \text { más frecuentes: } 13,0-13,9 \mathrm{~mm} \text { y } 14,0-14,9 \\
\mathrm{~mm} \\
\text { Los } 2 \text { rangos menos frecuentes: } 10,0-10,9 \mathrm{~mm} \text { y } 18,0-18,2 \mathrm{~mm}\end{array}$ \\
\hline Categorías del tamaño (longitud) de los ejemplares $(\mathrm{n}=59)$ & $\begin{array}{l}\text { Chicos: } 13 \text { ejemplares }(22,0 \%) \text { cuyos valores están entre } 10,3 \text { y } 12,9 \\
\text { mm } \\
\text { Medianos: } 31 \text { ejemplares }(52,5 \%) \text { cuyos valores están entre } 13,0 \text { y } \\
\text { 15,6 mm } \\
\text { Grandes: } 15 \text { ejemplares }(24,5 \%) \text { cuyos valores están entre } 15,7 \text { y } 18,2 \\
\text { mm }\end{array}$ \\
\hline $\begin{array}{l}\text { Número de puntuaciones en cada hilera central del pronoto } \\
(\mathrm{n}=59)\end{array}$ & $\begin{array}{l}5 \text { puntuaciones en cada hilera: } 48 \text { ejemplares }(81,4 \%) \text {. } \\
\text { Más de } 5 \text { puntuaciones en cada hilera: } 11 \text { ejemplares }(18,6 \%) \text {. }\end{array}$ \\
\hline Longitud del edéago $(\mathrm{n}=50)$ & $\begin{array}{l}\text { Promedio: } 1,50 \mathrm{~mm} \\
\text { Rango: } 1,25-1,95 \mathrm{~mm}\end{array}$ \\
\hline $\begin{array}{l}\text { Proporción longitud del edéago respecto a la longitud del } \\
\text { cuerpo }(n=50)\end{array}$ & $\begin{array}{l}\text { Promedio: } 10,5 \% \\
\text { Rango: } 8,6-13,4 \% .\end{array}$ \\
\hline $\begin{array}{l}\text { Categorías del tamaño de los edéagos (longitud) en } \\
\text { proporción a la longitud del cuerpo }(n=50)\end{array}$ & $\begin{array}{l}\text { Chicos: } 18 \text { ejemplares ( } 36 \% \text { ) cuyos valores de proporción están entre } \\
8,6 \text { y } 10,1 \% \text {. } \\
\text { Medianos: } 26 \text { ejemplares }(52 \%) \text { cuyos valores de proporción están } \\
\text { entre } 10,2 \text { y } 11,8 \% \text {. } \\
\text { Grandes: } 6 \text { ejemplares ( } 12 \% \text { ) cuyos valores de proporción están } \\
\text { entre } 11,0 \text { y } 13,4 \% \text {. }\end{array}$ \\
\hline PARÁmETROS EN HEMBRAS $(\mathrm{N}=58)$ & VALORES OBTENIDOS \\
\hline Longitud total del cuerpo & $\begin{array}{l}\text { Promedio: } 13,93 \mathrm{~mm} \\
\text { Rango: } 10,5-21,5 \mathrm{~mm} \\
\text { En rangos de } 1 \mathrm{~mm} \text {, los } 2 \text { más frecuentes: } 13,0-13,9 \text { y } 12,0-12,9 \mathrm{~mm} \\
\text { Los } 2 \text { rangos menos frecuentes: } 10,0-10,9 \text { y } 19,0-19,9 \mathrm{~mm}\end{array}$ \\
\hline Categorías del tamaño (longitud) de los ejemplares & $\begin{array}{l}\text { Chicos: } 36 \text { ejemplares }(62,1 \%) \text { cuyos valores están entre } 10,50 \text { y } \\
\text { 14,16 mm. } \\
\text { Medianos: } 16 \text { ejemplares }(27,6 \%) \text { cuyos valores están entre } 14,17 \text { y } \\
\text { 17,83 mm. } \\
\text { Grandes: } 6 \text { ejemplares }(10,3 \%) \text { cuyos valores están entre } 17,84 \text { y } \\
21,50 \mathrm{~mm} .\end{array}$ \\
\hline Número de puntuaciones en cada hilera central del pronoto & $\begin{array}{l}5 \text { puntuaciones en cada hilera: } 48 \text { ejemplares }(82,8 \%) \text {. } \\
\text { Más de } 5 \text { puntuaciones en cada hilera: } 10 \text { ejemplares }(17,2 \%) \text {. }\end{array}$ \\
\hline
\end{tabular}




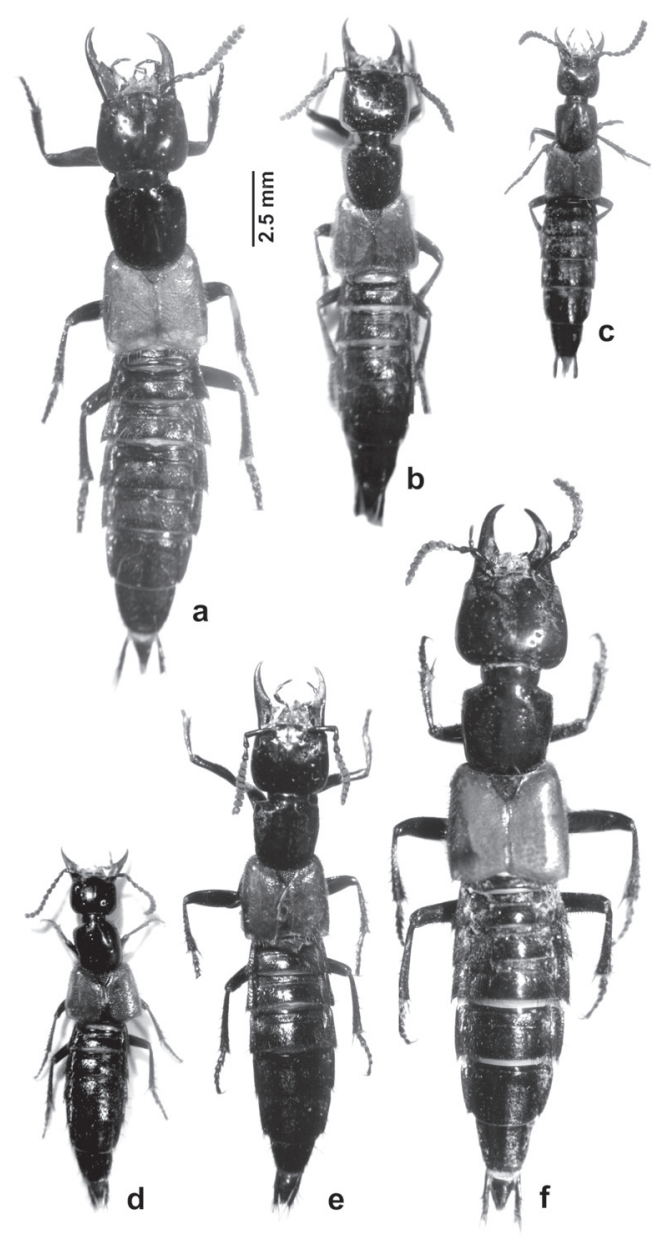

Figura 1. Variación de longitud total en Belonuchus ephippiatus. Machos: a) grande, b) mediano y c) chico). Hembras: d) chica, e) mediana y f) grande. / Variation of the total length in Belonuchus ephippiatus. Males: a) big, b) medium, c) small. Females: d) small, e) medium, f) big.

Longitud total $17,0 \mathrm{~mm}$. Cuerpo completamente negro, excepto los tarsos, palpos maxilares y el terguito genital que son café-rojizos, y el esternito genital que es amarillo en la base y café en el ápice.

Cabeza (Fig. 3b): ligeramente transversa (proporción largo/ancho $=0,92) ; 1,22$ veces más ancha que el pronoto. Superficie dorsal con microescultura en forma de líneas onduladas y puntuaciones setíferas dispersas; con una línea media longitudinal lisa poco visible. Puntuaciones interoculares medias separadas entre sí por casi 2 veces la distancia que las separa de las puntuaciones laterales. Ojos pequeños $(0,6 \mathrm{~mm}), 0,21$ veces tan largos como la cabeza. Antenómeros 1-3 alargados, 4-5 casi tan largos como anchos, antenómeros 6-10 transversos y el apical más pequeño que los anteriores. Labro bilobulado, cada lóbulo con una seda larga, tres de tamaño medio y siete

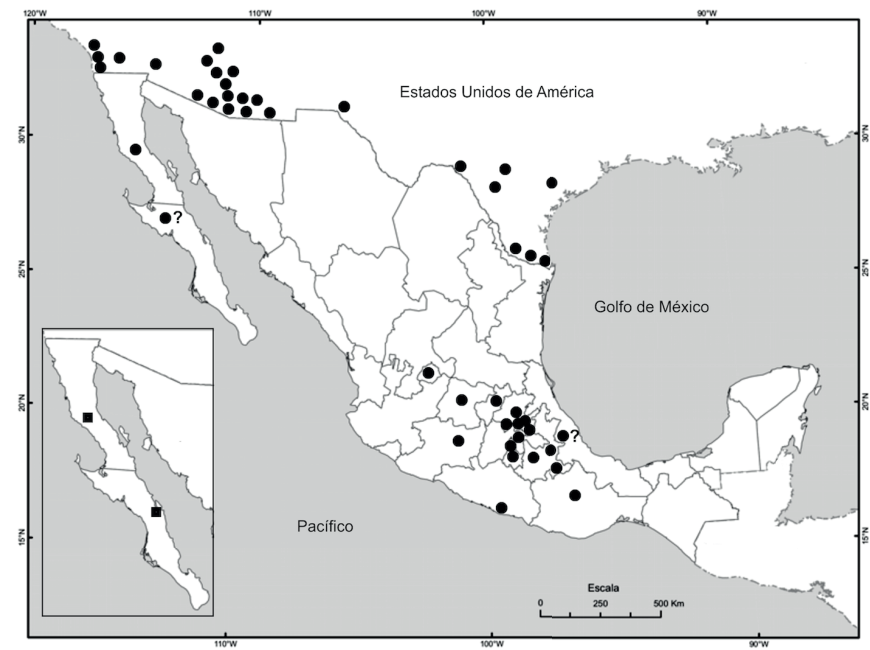

Figura 2. Distribución geográfica de B. ephippiatus (círculos negros) en el sur de E.U.A. y en México, y de B. moroni en la Península de Baja California (cuadros negros en el recuadro). Los datos fueron tomados de los ejemplares analizados y registros publicados (Sharp 1885, 1887; Fauvel 1901; Herman 2001; Navarrete-Heredia et al. 2002; Márquez \& Asiain 2006). Los signos de interrogación al lado del círculo negro indican que se desconoce la localidad precisa en ese estado. / Geographic distribution of B. ephippiatus (black circles) in the south of USA and Mexico, and of B. moroni in the Baja California Peninsula (black squares in the box). Data were taken of the analyzed specimens and published records (Sharp 1885, 1887; Fauvel 1901; Herman 2001; Navarrete-Heredia et al. 2002; Márquez \& Asiain 2006). Question marks next to the black circle indicate that the precise location in that state is unknown.

sedas cortas. Mandíbulas 3,25 mm de longitud que equivale a 1,2 veces la longitud de la cabeza; la izquierda con dos dientes cercanos que forman una " $U$ ", la derecha con un solo diente. Palpomero apical maxilar 1,35 veces tan largo como el palpomero preapical; palpomero apical labial 1,22 veces tan largo como la longitud del palpomero preapical. Superficie ventral cefálica lustrosa, con microescultura de líneas onduladas, y con sedas dispersas en las esquinas posteriores.

Tórax: pronoto casi tan largo como ancho (proporción $=$ $0,98)$ y 1,16 veces más ancho en las esquinas anteriores que en las posteriores; hileras centrales formadas por 5 puntuaciones cada una; microescultura como en la cabeza; bordes laterales con puntuaciones densas que originan macrosedas. Élitros con superficie lisa, cubiertos con sedas finas y algunas sedas largas; bordes laterales con macrosedas 
densas. Escutelo con microescultura de líneas onduladas combinadas con sedas finas. Zona central del prosternón, entre las procoxas, con sedas finas y esparcidas, así como dos sedas largas. Mesosternón con microescultura de líneas onduladas y algunas sedas finas; con una carena mediaposterior bien desarrollada. Metasternón con microescultura como el mesosternón, pero con sedas cortas más densas. Patas anteriores con la coxa cubierta de sedas densas en su superficie interna y sedas dispersas en la superficie externa; margen interno del fémur con dos hileras de espinas, la hilera anterior desarrollada desde la base hasta el ápice y la hilera posterior desde la base hasta el tercio anterior (Fig. 3c); tibia con espinas y sedas finas más densas que las tibias de las patas medias y posteriores, con 2 espolones apicales de color café. Tarsos de los tres pares de patas un tanto robustos, dorsalmente un poco aplanados y excavados, menos anchos que el ápice de la tibia, quinto tarsómero el más largo, seguido del primero, segundo, tercero y el cuarto el más pequeño. Coxa y fémur de las patas medias con sedas

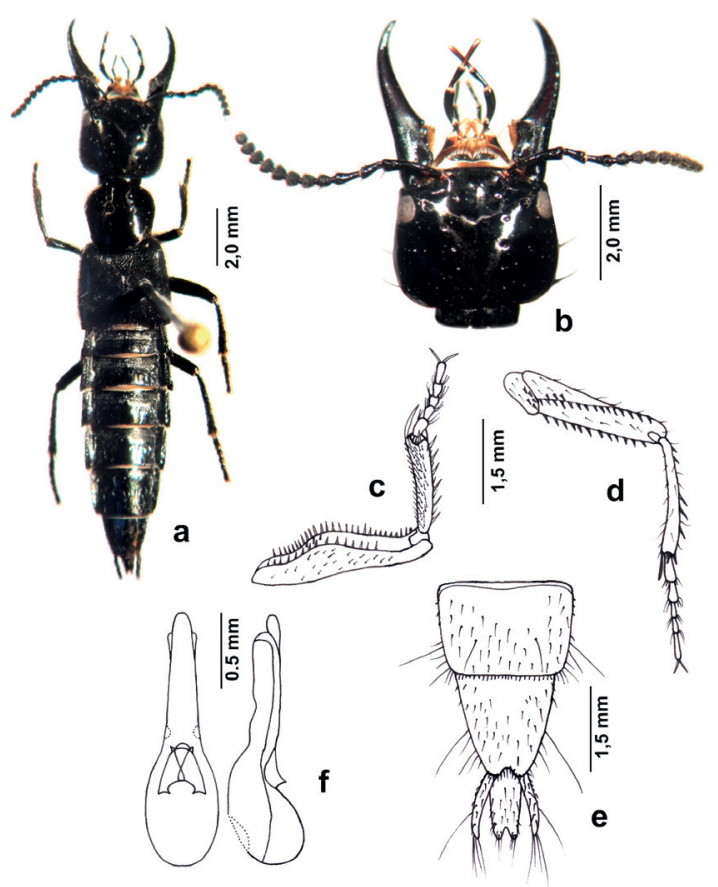

FIGURA 3. Belonuchus moroni sp. nov., a) vista dorsal de un paratipo (hembra), b) detalles de la cabeza, c) pata anterior izquierda mostrando las hileras de espinas en el fémur, d) pata posterior izquierda mostrando las hileras de espinas en el fémur, e) últimos tres esternitos abdominales del macho con los bordes anteriores del pregenital y genital escotados, f) vista ventral (izquierda) y lateral (derecha) del edeago. / Belonuchus moroni sp. nov., a) dorsal view of a paratype (female), b) details of the head, c) first left leg showing the rows of spines in the femur, d) posterior left leg showing the rows of spines in the femur, e) last three abdominal sternites of the male with emarginated anterior border of pregenital and genital segments, f) ventral (left) and lateral view (right) of the aedeagus. dispersas; tibia con sedas que son más densas en el tercio anterior del lado interno; ápice y lado interno del primer tarsómero con sedas gruesas densas, resto de los tarsómeros con sedas gruesas menos densas presentes sólo en el ápice. Coxa y fémur del tercer par de patas con sedas finas más densas que en la tibia y los tarsos; superficie interna del fémur con dos hileras de espinas gruesas en toda su longitud (Fig. 3d); tibia con pocas sedas finas y algunas espinas, ápice con varios espolones rojizos.

Abdomen: lustroso, con sedas finas dispersas que son más densas hacia los bordes laterales. Primeros tres terguitos visibles con una línea transversa impresa cerca del borde anterior; terguitos 1-4 con microescultura en forma de malla en el borde anterior. Esternito pre-genital escotado en su ápice (Fig. 3e). Esternito genital alargado; margen apical profundamente escotado en forma de "V" (Fig. 3e).

Edéago: longitud total 1,6 $\mathrm{mm}$; alargado, con la base del lóbulo medio convexa en vista lateral, y los parámeros fusionados y rectos en esa misma vista; ápice del lóbulo medio redondeado (Fig. 3f).

Variación (todos los paratipos son hembras): longitud total $14,8-17,8 \mathrm{~mm}$. Proporción largo/ancho de la cabeza $=0,86-$ 0,97; cabeza 1,03-1,17 veces más ancha que el pronoto. Mandíbulas 1,0-1,24 veces tan larga como la cabeza. Proporción largo/ancho del pronoto $=0,92-0,98$. Proporción de la anchura anterior (ángulos anteriores) / anchura posterior (ángulos posteriores) del pronoto $=1,16-1,21$. Longitud de los élitros $=2,8-3,5 \mathrm{~mm}$. Pronoto con 5 a 6 puntuaciones en cada hilera central del pronoto, con el par posterior más separado del par anterior contiguo que la separación entre los tres pares anteriores. Cuarto terguito abdominal visible con la línea impresa transversa interrumpida en su parte media en cuatro ejemplares hembras y en la quinta hembra no se aprecia esta línea.

Diagnosis: la única especie con la cual se puede confundir a $B$. moroni es B. ephippiatus, pero B. moroni es completamente negra, la línea lisa longitudinal de la cabeza está poco visible, las mandíbulas son más largas que la cabeza $(1,04$ a 1,20 veces), el palpómero maxilar apical es notablemente más largo que el preapical (1,25 a 1,35 veces) (Fig. 3b), el abdomen posee menor densidad de sedas en comparación con B. ephippiatus, y el edéago tiene el ápice del lóbulo medio redondeado (Fig. 3f); mientras que B. ephippiatus tiene los élitros anaranjados (el resto del cuerpo es negro), la línea lisa longitudinal de la cabeza es claramente visible, las mandíbulas son más cortas o casi tan largas como la longitud de la cabeza (0,82 a 1,06 veces) (Fig. 1), el palpómero apical maxilar es ligeramente más largo que el preapical $(1,00 \mathrm{a}$ 1,25 veces, $\mathrm{n}=20$ ), el abdomen posee mayor densidad de sedas y el edéago tiene el ápice del lóbulo medio alargado. 
Etimología. Nos da gusto dedicar el nombre de esta especie al Dr. Miguel Ángel Morón Ríos, recientemente fallecido, en reconocimiento a su importante contribución al estudio de los insectos (particularmente de Scarabaeoidea), así como por sus enseñanzas y su apreciada amistad hacia nosotros.
Distribución geográfica. Esta especie se registra de La Sepultura, Ensenada, Baja California, y de Armenta, Loreto, Baja California Sur (Fig. 2). Estas localidades pueden ser ubicadas como pertenecientes a la provincia biogeográfica de Baja California (Morrone 2006).

Tabla 3. Medidas de longitud total del cuerpo (LT) y número de puntuaciones en cada hilera central del pronoto de 58 hembras de $B$. ephippiathus y/o B. erythropterus. Entre paréntesis se anota si se trata de ejemplares chicos (CH), medianos (ME) o grandes (GR). I Measurements of total body length (LT) and number of punctures in each central row of the pronotum of 58 females of Belonuchus ephippiathus and/or B. erythropterus. In parentheses it is written whether the specimens are small (CH), medium (ME) or large (GR) specimens.

\begin{tabular}{|c|c|c|}
\hline Estado / Municipio (localidad) & $\begin{array}{l}\text { Longitud total del cuerpo } \\
\text { (LT) }(\mathrm{mm})\end{array}$ & $\begin{array}{l}\text { \# de puntuaciones en } \\
\text { cada hilera central del } \\
\text { pronoto diferente a 5:5 }\end{array}$ \\
\hline 1. Aguascalientes / San José de Gracia (Agua Zarca) & $11,1(\mathrm{CH})$ & \\
\hline 2. Baja California / Ensenada (Cataviña) & $11,3(\mathrm{CH})$ & \\
\hline 3. Baja California / Ensenada (Cataviña) & $11,0(\mathrm{CH})$ & \\
\hline 4. Baja California / Ensenada (Cataviña) & $12,6(\mathrm{CH})$ & \\
\hline 5. Baja California / Ensenada (Cataviña) & $11,8(\mathrm{CH})$ & \\
\hline 6. Baja California / Ensenada (Cataviña) & $12,1(\mathrm{CH})$ & \\
\hline 7. Hidalgo / Acatlán (Loma Larga) & $15,1(\mathrm{ME})$ & \\
\hline 8. Hidalgo / Actopan (camino a La Magdalena) & $13,9(\mathrm{CH})$ & \\
\hline 9. Hidalgo / Atotonilco El Grande & $19,0(\mathrm{GR})$ & $5: 6$ \\
\hline 10. Hidalgo / Huasca de Ocampo (San Miguel Regla) & $13,8(\mathrm{CH})$ & \\
\hline 11. Hidalgo / Huasca de Ocampo (Santa Ma. Regla) & $18,8(\mathrm{GR})$ & $6: 6$ \\
\hline 12. Hidalgo / Metztitlán (RBBM) & $13,2(\mathrm{CH})$ & $5: 6$ \\
\hline 13. Hidalgo / Metztitlán (RBBM) & $13,4(\mathrm{CH})$ & \\
\hline 14. Hidalgo / Metztitlán (RBBM) & $14,2(\mathrm{ME})$ & \\
\hline 15. Hidalgo / Metztitlán (RBBM) & $12,1(\mathrm{CH})$ & \\
\hline 16. Hidalgo / Pachuca (Cubitos) & $14,0(\mathrm{CH})$ & \\
\hline 17. Hidalgo / Santiago de Anaya (El Sitio) & 21,5 (GR) & 9:9 \\
\hline 18. Hidalgo / Singuilucan (Matías Rodríguez) & $13,5(\mathrm{CH})$ & \\
\hline 19. Hidalgo / Tolcayuca (cerro Mambrú) & $18,5(\mathrm{GR})$ & $5: 6$ \\
\hline 20. Hidalgo / Zempoala (Sierra de Pitos) & $15,1(\mathrm{ME})$ & \\
\hline 21. Hidalgo / Zempoala (Sierra de Pitos) & $14,8(\mathrm{ME})$ & $7: 7$ \\
\hline 22. Hidalgo / Zempoala (La Trinidad) & $18,6(\mathrm{GR})$ & \\
\hline 23. Morelos / Tlayacapan (camino a Tlalnepantla) & $13,6(\mathrm{CH})$ & \\
\hline 24. Morelos / Tlayacapan (camino a Tlalnepantla) & $14,0(\mathrm{CH})$ & \\
\hline 25. Morelos / Tlayacapan (camino a Tlalnepantla) & $12,4(\mathrm{CH})$ & \\
\hline 26. Morelos / Tlayacapan (camino a Tlalnepantla) & $12,2(\mathrm{CH})$ & \\
\hline 27. Morelos / Tlayacapan (camino a Tlalnepantla) & $12,6(\mathrm{CH})$ & \\
\hline 28. Morelos / Tlayacapan (camino a Tlalnepantla) & $11,8(\mathrm{CH})$ & \\
\hline 29. Morelos / Tlayacapan (camino a Tlalnepantla) & $12,2(\mathrm{CH})$ & \\
\hline 30. Morelos / Tlayacapan (camino a Tlalnepantla) & $10,5(\mathrm{CH})$ & \\
\hline 31. Morelos / Tlayacapan (camino a Tlalnepantla) & $13,1(\mathrm{CH})$ & \\
\hline 32. Morelos / Tlayacapan (camino a Tlalnepantla) & $12,3(\mathrm{CH})$ & \\
\hline 33. Puebla / San Nicolás de Buenos Aires (carretera a Xalapa, km 63) & $16,7(\mathrm{ME})$ & $6: 6$ \\
\hline 34. Querétaro / Bernal (Peña) & $14,7(\mathrm{ME})$ & \\
\hline 35. Querétaro / Bernal (Peña) & $12,0(\mathrm{CH})$ & \\
\hline 36. Querétaro / Bernal (Peña) & $14,9(\mathrm{ME})$ & \\
\hline 37. Querétaro / Bernal (Peña) & $16,7(\mathrm{ME})$ & \\
\hline
\end{tabular}


Taxonomía de Belonuchus grupo ephippiatus: MÁRQuEZ, J. ET AL.

\begin{tabular}{|c|c|c|}
\hline Estado / Municipio (localidad) & $\begin{array}{l}\text { Longitud total del cuerpo } \\
\text { (LT) }(\mathrm{mm})\end{array}$ & $\begin{array}{l}\text { \# de puntuaciones en } \\
\text { cada hilera central del } \\
\text { pronoto diferente a 5:5 }\end{array}$ \\
\hline 38. Querétaro / Bernal (Peña) & 19,5 (GR) & $5: 6$ \\
\hline 39. Querétaro / Bernal (Peña) & $11,5(\mathrm{CH})$ & \\
\hline 40. Querétaro / Bernal (Peña) & $12,2(\mathrm{CH})$ & \\
\hline 41. Querétaro / Bernal (Peña) & $13,2(\mathrm{CH})$ & \\
\hline 42. Querétaro / Bernal (Peña) & $14,7(\mathrm{ME})$ & \\
\hline 43. Querétaro / Bernal (Peña) & $11,8(\mathrm{CH})$ & \\
\hline 44. Querétaro / Bernal (Peña) & $15,2(\mathrm{ME})$ & \\
\hline 45. Querétaro / Bernal (Peña) & $16,5(\mathrm{ME})$ & $6: 6$ \\
\hline 46. Querétaro / Bernal (Peña) & $15,7(\mathrm{ME})$ & \\
\hline 47. Querétaro / Bernal (Peña) & $13,6(\mathrm{CH})$ & \\
\hline 48. Querétaro / Bernal (Peña) & $10,7(\mathrm{CH})$ & $6: 5$ \\
\hline 49. Querétaro / Bernal (Peña) & $11,8(\mathrm{CH})$ & \\
\hline 50. Querétaro / Bernal (Peña) & $14,2(\mathrm{ME})$ & \\
\hline 51. Querétaro / Bernal (Peña) & $13,0(\mathrm{CH})$ & \\
\hline 52. Querétaro / Bernal (Peña) & $14,7(\mathrm{ME})$ & \\
\hline 53. Querétaro / Bernal (Peña) & $13,9(\mathrm{CH})$ & \\
\hline 54. Querétaro / Bernal (Peña) & $12,2(\mathrm{CH})$ & \\
\hline 55. Querétaro / Bernal (carretera Bernal-Tolimán, km 44) & $11,7(\mathrm{CH})$ & \\
\hline 56. Querétaro / Bernal (carretera Bernal-Tolimán, km 44) & $14,7(\mathrm{ME})$ & \\
\hline 57. Querétaro / Bernal (carretera Bernal-Tolimán, km 44) & $15,0(\mathrm{ME})$ & \\
\hline 58. Querétaro / Bernal (carretera Bernal-Tolimán, km 44) & $13,1(\mathrm{CH})$ & \\
\hline
\end{tabular}

TABLA 4. Análisis de la variación morfológica de machos y hembras de Belonuchus ephippiathus y/o B. erythropterus en las tres localidades mejor representadas en la muestra. / Analysis of the morphological variation of males and females of Belonuchus ephippiathus and/or B. erythropterus in the three localities best represented in the sample.

\begin{tabular}{|c|c|c|}
\hline LOCALIDADES & PARÁMETROS & VALORES OBTENIDOS \\
\hline \multirow[t]{8}{*}{$\begin{array}{l}\text { La Peña de Bernal, } \\
\text { Querétaro }(n=46)\end{array}$} & $\begin{array}{l}\text { MACHOS } \\
\text { Longitud total del cuerpo }(n=25)\end{array}$ & $\begin{array}{l}\text { MACHOS } \\
\text { Promedio: } 14,2 \mathrm{~mm} \\
\text { Rango: } 11,1-18,2 \mathrm{~mm}\end{array}$ \\
\hline & Categorías del tamaño de los ejemplares $(\mathrm{N}=25)$ & $\begin{array}{l}\text { Chicos: } 7 \text { ejemplares. } \\
\text { Medianos: } 13 \text { ejemplares. } \\
\text { Grandes: } 5 \text { ejemplares. }\end{array}$ \\
\hline & $\begin{array}{l}\text { Número de puntuaciones en cada hilera central del } \\
\text { pronoto }(\mathrm{n}=25)\end{array}$ & $\begin{array}{l}5 \text { puntuaciones en cada hilera: } 22 \text { ejemplares } \\
\text { Más de } 5 \text { puntuaciones en cada hilera: } 3 \text { ejemplares. }\end{array}$ \\
\hline & $\begin{array}{l}\text { Proporción longitud del edéago respecto a la } \\
\text { longitud del cuerpo }(\mathrm{n}=22)\end{array}$ & $\begin{array}{l}\text { Promedio: } 9,8 \% \\
\text { Rango: } 8,8-12,0 \%\end{array}$ \\
\hline & $\begin{array}{l}\text { Categorías del tamaño de los edéagos (longitud) } \\
\text { en proporción a la longitud del cuerpo }(n=22)\end{array}$ & $\begin{array}{l}\text { Chicos: } 9 \text { ejemplares } \\
\text { Medianos: } 11 \text { ejemplares } \\
\text { Grandes: } 1 \text { ejemplar }\end{array}$ \\
\hline & $\begin{array}{l}\text { HEMBRAS }(\mathrm{n}=21) \\
\text { Longitud total del cuerpo }\end{array}$ & $\begin{array}{l}\text { HEMBRAS } \\
\text { Promedio: } 13,94 \mathrm{~mm} \\
\text { Rango: } 10,7-19,5 \mathrm{~mm}\end{array}$ \\
\hline & Categorías del tamaño de los ejemplares & $\begin{array}{l}\text { Chicos: } 11 \text { ejemplares. } \\
\text { Medianos: } 9 \text { ejemplares. } \\
\text { Grandes: } 1 \text { ejemplar. }\end{array}$ \\
\hline & $\begin{array}{l}\text { Número de puntuaciones en cada hilera central del } \\
\text { pronoto }\end{array}$ & $\begin{array}{l}5 \text { puntuaciones en cada hilera: } 18 \text { ejemplares } \\
\text { Más de } 5 \text { puntuaciones en cada hilera: } 3 \text { ejemplares. }\end{array}$ \\
\hline
\end{tabular}




\begin{tabular}{|c|c|c|}
\hline LOCALIDADES & PARÁmetros & VALORES OBTENIDOS \\
\hline \multirow[t]{8}{*}{$\begin{array}{l}\text { Barranca de } \\
\text { Metztitlán, Hidalgo }\end{array}$} & $\begin{array}{l}\text { MACHOS } \\
\text { Longitud total del cuerpo }(n=9)\end{array}$ & $\begin{array}{l}\text { MACHOS } \\
\text { Promedio: } 14,7 \mathrm{~mm} \\
\text { Rango: } 12,0-16,8 \mathrm{~mm}\end{array}$ \\
\hline & Categorías del tamaño de los ejemplares $(\mathrm{N}=9)$ & $\begin{array}{l}\text { Chicos: } 1 \text { ejemplar. } \\
\text { Medianos: } 5 \text { ejemplares. } \\
\text { Grandes: } 3 \text { ejemplares. }\end{array}$ \\
\hline & $\begin{array}{l}\text { Número de puntuaciones en cada hilera central del } \\
\text { pronoto }(n=9)\end{array}$ & $\begin{array}{l}5 \text { puntuaciones en cada hilera: } 6 \text { ejemplares } \\
\text { Más de } 5 \text { puntuaciones en cada hilera: } 3 \text { ejemplares. }\end{array}$ \\
\hline & $\begin{array}{l}\text { Proporción longitud del edéago respecto a la } \\
\text { longitud del cuerpo }(n=8)\end{array}$ & $\begin{array}{l}\text { Promedio: } 9,8 \% \\
\text { Rango: } 8,6-10,8 \%\end{array}$ \\
\hline & $\begin{array}{l}\text { Categorías del tamaño de los edéagos (longitud) } \\
\text { en proporción a la longitud del cuerpo }(n=8)\end{array}$ & $\begin{array}{l}\text { Chicos: } 6 \text { ejemplares } \\
\text { Medianos: } 2 \text { ejemplares } \\
\text { Grandes: } 0 \text { ejemplares }\end{array}$ \\
\hline & $\begin{array}{l}\text { HEMBRAS }(\mathrm{n}=4) \\
\text { Longitud total del cuerpo }\end{array}$ & $\begin{array}{l}\text { HEMBRAS } \\
\text { Promedio: } 13,2 \mathrm{~mm} \\
\text { Rango: } 12,1-14,2 \mathrm{~mm}\end{array}$ \\
\hline & Categorías del tamaño de los ejemplares & $\begin{array}{l}\text { Chicos: } 3 \text { ejemplares. } \\
\text { Medianos: } 1 \text { ejemplar. } \\
\text { Grandes: } 0 \text { ejemplar. }\end{array}$ \\
\hline & $\begin{array}{l}\text { Número de puntuaciones en cada hilera central del } \\
\text { pronoto }\end{array}$ & $\begin{array}{l}5 \text { puntuaciones en cada hilera: } 3 \text { ejemplares } \\
\text { Más de } 5 \text { puntuaciones en cada hilera: } 1 \text { ejemplar. }\end{array}$ \\
\hline \multirow[t]{8}{*}{$\begin{array}{l}\text { Sierra de Pitos, } \\
\text { Hidalgo }\end{array}$} & $\begin{array}{l}\text { MACHOS } \\
\text { Longitud total del cuerpo }(n=8)\end{array}$ & $\begin{array}{l}\text { MACHOS } \\
\text { Promedio: } 14,68 \mathrm{~mm} \\
\text { Rango: } 10,3-17,5 \mathrm{~mm}\end{array}$ \\
\hline & Categorías del tamaño de los ejemplares $(\mathrm{N}=8)$ & $\begin{array}{l}\text { Chicos: } 2 \text { ejemplar. } \\
\text { Medianos: } 2 \text { ejemplares. } \\
\text { Grandes: } 4 \text { ejemplares. }\end{array}$ \\
\hline & $\begin{array}{l}\text { Número de puntuaciones en cada hilera central del } \\
\text { pronoto }(n=8)\end{array}$ & $\begin{array}{l}5 \text { puntuaciones en cada hilera: } 6 \text { ejemplares } \\
\text { Más de } 5 \text { puntuaciones en cada hilera: } 2 \text { ejemplares. }\end{array}$ \\
\hline & $\begin{array}{l}\text { Proporción longitud del edéago respecto a la } \\
\text { longitud del cuerpo }(n=7)\end{array}$ & $\begin{array}{l}\text { Promedio: } 10,6 \% \\
\text { Rango: } 8,7-12,1 \%\end{array}$ \\
\hline & $\begin{array}{l}\text { Categorías del tamaño de los edéagos (longitud) } \\
\text { en proporción a la longitud del cuerpo }(n=7)\end{array}$ & $\begin{array}{l}\text { Chicos: } 2 \text { ejemplares } \\
\text { Medianos: } 4 \text { ejemplares } \\
\text { Grandes: } 1 \text { ejemplares }\end{array}$ \\
\hline & $\begin{array}{l}\text { HEMBRAS }(\mathrm{n}=2) \\
\text { Longitud total del cuerpo }\end{array}$ & $\begin{array}{l}\text { HEMBRAS } \\
\text { Promedio: } 13,2 \mathrm{~mm} \\
\text { Rango: } 14,8-15,1 \mathrm{~mm}\end{array}$ \\
\hline & Categorías del tamaño de los ejemplares & No se obtuvo porque sólo son 2 ejemplares. \\
\hline & $\begin{array}{l}\text { Número de puntuaciones en cada hilera central del } \\
\text { pronoto }\end{array}$ & $\begin{array}{l}5 \text { puntuaciones en cada hilera: } 1 \text { ejemplar } \\
\text { Más de } 5 \text { puntuaciones en cada hilera: } 1 \text { ejemplar. }\end{array}$ \\
\hline
\end{tabular}

\section{DISCUSIÓN}

De acuerdo con Smetana (1995), B. ephippiatus se distingue de $B$. erytrhopterus por ser de menor tamaño (de 6,8 a $12,0 \mathrm{~mm}$ ), de forma menos robusta, con la cabeza menos ancha hacia atrás y sus ángulos posteriores también poco ensanchados, la puntuación sobre la cabeza es en general más dispersa, las hileras centrales del pronoto están formadas generalmente por 5 puntuaciones cada una, los terguitos abdominales con puntuación más densa (aunque también señala que es muy variable desde poco hasta muy densa), y el edéago es similar al de $B$. erytrhropterus pero ligeramente más corto. Mientras que $B$. erytrhopterus es de mayor tamaño ( 8,0 a $16,4 \mathrm{~mm})$, más robusta, con la cabeza 
y los ángulos posteriores más ensanchados, la puntuación sobre la cabeza en general más densa, las hileras centrales del pronoto están formadas por 6 a 8 puntuaciones cada una, a veces irregularmente dispuestas, raramente con 5 puntuaciones, los terguitos abdominales con puntuación esparcida y fina, y el edéago ligeramente más largo que el de B. ephippiatus.

De todas las diferencias anteriores, Smetana (1995) destaca la referente al número de puntuaciones en cada hilera central del pronoto, ya que ante la ausencia del material tipo de B. erythropterus de Solsky, sólo le fue posible reconocer en la descripción original este detalle que hace mención a las 6 o más puntuaciones en esta última especie. Con base en nuestras observaciones, la forma más robusta del cuerpo y cabeza más ancha hacia atrás están relacionadas con los ejemplares de tamaño mediano y grande, son características relacionadas con el tamaño del ejemplar que pueden considerarse como variación de una sola especie en lugar de dos. En cuanto a la densidad de puntuaciones en la superficie dorsal de la cabeza, no se apreciaron diferencias que pudieran ser cuantificadas. Con relación a la densidad de puntuaciones en los terguitos abdominales, el propio Smetana (1995) señala la gran variación que se presenta en $B$. ephippiatus que incluso pudo ser la causa de que se hayan descrito varios nombres sinónimos de esta especie en el pasado. Por lo anterior, no es de utilidad para separar a las dos posibles especies.

Las diferencias fundamentales entre las especies analizadas involucran la longitud del cuerpo, el número de puntuaciones en las hileras centrales del pronoto y la longitud del edéago. Con relación a la longitud total del cuerpo, es notable que los más de 600 ejemplares de B. ephippiatus revisados por Smetana (1995) posean una longitud marcadamente menor a los analizados en este trabajo, ésta es de 6,8 a 12,0 mm contra 10,3 a 18,2 en los machos y 10,5 a $21,5 \mathrm{~mm}$ en las hembras de nuestro análisis. En el caso de los 21 ejemplares de B. erythropterus estudiados por Smetana (1995), éstos tienen una longitud de 8,0 a 16,4 $\mathrm{mm}$, la cual se aproxima más a los organismos analizados aquí. Desconocemos cuál es la causa de esa menor longitud en los ejemplares de B. ephippiatus analizados por Smetana, que incluso es menor que nuestros organismos considerados como pequeños $(10,3$ a $12,9 \mathrm{~mm})$, tampoco se especifica a partir de cuántos ejemplares y de qué sexo se obtuvo ese rango. Sin embargo, los ejemplares que hemos analizado cumplen con todas las características del grupo ephippiatus propuesto por Smetana (1995), y hasta ahora que se describe aquí una especie nueva de este grupo, no conocemos otra con la que se puedan confundir.

Una posible causa de las diferencias en longitud del cuerpo que se comentó en el párrafo anterior, es la preparación de los ejemplares para su medición, pues deben montarse lo más recto posible y el edéago en los machos debe ser disecado para poder medirlo. En organismos que no se estiran cuando se montan, el abdomen queda más corto y puede conducir a una medición menor de longitud total. Una segunda posibilidad es que la variación tan amplia que se ha observado en esta especie incluya organismos más pequeños, al menos en el sur de E.U.A.

Las tres categorías de la longitud de los organismos sugieren que hay dominancia de aquellos de tamaño mediano y son menos comunes los de tamaño chico y grande. El hecho de separar dos especies sólo por el tamaño nos conduciría a tener al menos tres especies, porque después de los organismos medianos, es casi tan probable encontrar organismos grandes como chicos (Fig. 1a-c). En el caso de las hembras el rango se amplió más que en los machos porque se encontró al menos una hembra más pequeña y una muy grande que causó este efecto, pero se denota en esta parte del análisis que la variación de tamaño no es un carácter de dimorfismo sexual (Fig. 1d-f).

Tanto en machos como en hembras se presentaron organismos con 6 puntuaciones o más en cada hilera central del pronoto y casi en la misma proporción con respecto al total analizado (17 a $18 \%$ ). Los datos indican que el mayor número de puntuaciones se relaciona con ejemplares de tamaño mediano y grande, pero no son exclusivos. No todos los ejemplares de tamaño grande tienen más puntuaciones en las hileras centrales del pronoto, por lo que no es un carácter útil para separar a las dos especies, porque además esta variación se presenta en ejemplares de tamaño mediano y excepcionalmente en organismos chicos.

Con relación a la longitud del edéago, los ejemplares de tamaño grande poseen edéagos de mayor longitud que los medianos y chicos. Sin embargo, al considerar la proporción que representa la longitud del edéago respecto a la longitud del cuerpo de cada organismo, se apreció que en la mayoría de los ejemplares de tamaño chico y mediano sus edéagos son de tamaño mediano, con menor frecuencia poseen edéagos chicos y grandes. Por su parte, los ejemplares de tamaño grande cuentan con edéagos chicos o medianos, ninguno fue grande en proporción al cuerpo. Por lo anterior, la longitud del edéago en proporción a la longitud del cuerpo no es útil para separar los ejemplares estudiados en dos especies. Además de la variación en longitud del edéago, no apreciamos otras diferencias que pudieran ser útiles para separar dos especies. El esquema del edéago de B. ephippiatus y B. erythropterus se puede consultar en el trabajo de Smetana (1995, páginas 910 y 920 respectivamente).

El análisis de las tres localidades mejor representadas en la muestra conduce a las mismas conclusiones que al analizar toda la muestra, lo que sugiere que no hay efecto geográfico en los caracteres analizados y por lo tanto las conclusiones pueden aplicarse a cualquier área geográfica donde se registre la especie o al menos a las de procedencia de los organismos estudiados.

A pesar de no haber analizado el lectotipo designado 
por Smetana (1995) para B. ephippiatus, consideramos que nuestro análisis cuenta con suficiente robustez para proponer que B. ephippiatus y B. erythropterus son una sola especie, con la segunda de ellas como sinónima junior de la primera. Además, se considera que los principales caracteres propuestos previamente para diferenciarlas son parte de una marcada variación morfológica de esta especie. De esta manera, por ahora el grupo ephippiatus de Belonuchus está integrado por B. ephippiatus y B. moroni n. sp. Nuestra propuesta de sinonimia es la segunda, debido a que Fauvel (1901) la había propuesto previamente y fue hasta 1995 que Smetana revalidó a $B$. erythropterus con la misma carencia que nosotros de no haber podido localizar y estudiar los tipos de esas especies. Desconocemos si Fauvel consultó o no los tipos involucrados, pero nuestras conclusiones apoyan su propuesta.

Por lo tanto, la distribución geográfica de B. ephippiatus incluye 13 estados Mexicanos y existe una carencia notable de registros en los estados del norte de México, en donde consideramos que es altamente probable su existencia, por ejemplo en Chihuahua, Sonora, Durango, Coahuila, Zacatecas, entre otros (Fig. 2). La mayor cantidad de registros están en la provincia biogeográfica del Altiplano Mexicano, la cual se extiende desde el sur de E.U.A. e incluye porciones extensas, áridas y semiáridas, de México hasta su confluencia al sur con el Eje Volcánico Transmexicano, al oriente y occidente con las respectivas Sierras Madres, con las cuales comparte algunos registros en sus zonas de confluencia. Una parte de la distribución se ubica al sur del Eje Volcánico Transmexicano, en las zonas xerófilas o bosques con presencia de cactáceas y magueyes de Puebla y Oaxaca. Dicha distribución hace interesante plantear un análisis biogeográfico, posiblemente filogeográfico, encaminado a conocer el efecto del Eje Volcánico Transmexicano en la distribución geográfica de esta especie y otras con patrones de distribución similar. Los registros publicados de B. ephippiatus para Colombia y Venezuela (Fauvel 1901, Herman 2001, Navarrete-Heredia et al. 2002) requieren verificación, ya que se trata de una distribución atípica que sugiere la posibilidad de que se trate de una especie diferente.

Con base en la gran variación morfológica encontrada en B. ephippiatus, se deduce que esta especie es depredadora, con gran preferencia por buscar presas en plantas suculentas, principalmente cactáceas y magueyes, que se transforman en un microhábitat importante en sitios con condiciones de sequía (extrema en ocasiones), pues al entrar en etapa de descomposición, albergan gran cantidad de larvas, principalmente de dípteros, además de contar con agua (o al menos tejidos bien hidratados) y ofrecer refugio sombreado a diversas especies, entre ellas B. ephippiatus, así como otras especies de estafilínidos, histéridos, tenebriónidos, cerambícidos, entre otros grupos. Probablemente la disponibilidad de cactáceas y magueyes en descomposición, y las presas potenciales que ahí se encuentren, sea muy variable a lo largo del extenso territorio que ocupan y también dependiendo de la época del año, lo que a su vez pudiera afectar el tamaño del cuerpo y otras estructuras (por ejemplo el número de puntuaciones en el pronoto y la longitud del edéago) de los ejemplares de $B$. ephippiatus durante su desarrollo larval hasta convertirse en adultos. Los datos de las tres localidades mejor representadas (Tabla 4) sugieren que la variación comentada se da tanto dentro de una misma población como entre diferentes poblaciones.

Por su parte, B. moroni se conoce por ahora sólo de una localidad de Baja California (Ensenada, La Sepultura) y una de Baja California Sur (Loreto, Armenta), asociada a la cactácea columnar conocida como "senita" (Lophocereus schottii) (Pfeiler et al. 2013). Al parecer posee hábitos de vida y alimenticios similares a $B$. ephippiatus, pero su distribución podría estár limitada a la provincia biogeográfica de Baja California (sensu Morrone 2006); sin embargo, hacen falta más muestreos para conocer mejor estos aspectos.

\section{AGRADECIMIENTOS}

Agradecemos a E. Pfeiler (Centro de Investigación en Alimentación y Desarrollo, A.C., Guaymas, Sonora) por la generosa donación de la serie tipo de B. moroni, así como a dos revisores anónimos por las valiosas sugerencias aportadas para la mejora de este trabajo.

\section{REFERENCIAS}

Asiain, J., Márquez, J., Bueno-Villegas, J. 2011. The Staphylinidae (Coleoptera) Fauna of Los Mármoles National Park, Hidalgo, Mexico. The Coleopterists Bulletin 65(4):393402.

Fauvel, A. 1901. Voyage de M. le Dr. Ed. Bugnion au Venezuela, en Colombie et aux Antilles. Revue d'Entomologie 20:6991.

Herman, L. 2001. Catalog of the Staphylinidae (Insecta: Coleoptera). 1758 to the end of the second millennium. V. Staphylinine Group (Part 2). Staphylininae: Diochini, Maorothiini, Othiini, Platyprosopini, Staphylinini (Amblyopinina, Anisolinina, Hyptiomina, Philonthina). Bulletin of the American Museum of Natural History 265:2441-3020.

Huerta Camacho, A.Z. 2010. Diversidad de coleópteros necrófilos (Insecta: Coleoptera) en un matorral xerófilo de Epazoyucan y un bosque de pino-encino de Singuilucan, Hidalgo, México. Tesis profesional, Licenciatura en Biología, UAEH. Mineral de la Reforma, Hidalgo. 81 pp.

JiMÉnEz SÁnchez, E. 1998. Estafilínidos (Coleoptera: Staphylinidae) necrófilos de la Sierra de Nanchititla, Estado de México. Tesis profesional, Licenciatura en Biología, Escuela Nacional de Estudios Profesionales Iztacala, UNAM. 
México, D.F. 97 pp.

MÁrqueZ, J. 2001. Especies necrófilas de Staphylinidae (Insecta: Coleoptera) del municipio de Tlayacapan, Morelos, México. Folia Entomológica Mexicana 40(1):93-131.

MÁrQueZ, J. 2003. Ecological patterns on necrophilous Staphylinidae (Insecta: Coleoptera) from Tlayacapan, Morelos, México. Acta Zoológica Mexicana (n. s.) 89:69-83.

Márquez, J. 2004. Primeros registros estatales de especies mexicanas de Staphylininae (Coleoptera: Staphylinidae). Acta Zoológica Mexicana (n. s.) 20(1):91-97.

Márquez, J. 2006. Primeros registros estatales y datos de distribución geográfica de especies mexicanas de Staphylinidae (Coleoptera). Boletín Sociedad Entomológica Aragonesa 38:181-198.

Márquez, J., Asiain, J. 2006. Patrones de distribución de la familia Staphylinidae (Coleoptera). En: Morrone, J.J., Llorente Bousquets, J. (Eds.) Componentes bióticos principales de la Entomofauna Mexicana: 157-236. Las Prensas de Ciencias, UNAM, México, D.F.

Márquez, J., Asiain, J. 2012. Primeros registros estatales de especies mexicanas de Staphylinidae (Insecta: Coleoptera). Acta Zoológica Mexicana (n. s.) 28(1):118-132.

Márquez, J., Asiain, J., Santiago-Jiménez, Q.J. 2004. Especies de Staphylininae (Coleoptera: Staphylinidae) de "El Mirador", Veracruz, México. Dugesiana 10(2):21-46.

Morrone, J.J. 2006. Biogeographic areas and transition zones of Latin America and the Caribbean Islands based on panbiogeographic and cladistic analyses of the entomofauna. Annual Review of Entomology 51:467-494.

Navarrete-Heredia, J.L. 1995. Aspectos biológicos de Philonthus apiciventris y P. oxyporinus (Coleoptera: Staphylinidae: Staphylininae), en una zona de Morelos, México, con una lista de las especies Mexicanas de Philonthus. Anales del Instituto de Biología, Universidad Nacional Autónoma de México, Serie Zoología 66(1):81-106.

Navarrete-Heredia, J.L. 1996. Coleópteros micetócolos de Basidiomycetes de San José de los Laureles, Morelos, México. Tesis profesional, Maestría en Ciencias (Biología Animal), Facultad de Ciencias, UNAM. México, D.F. $179 \mathrm{pp}$.

Navarrete-Heredia, J.L., Newton, A.F., Thayer, M.K., Ashe, J.S., Chandler, D.S. 2002. Guía ilustrada para los géneros de
Staphylinidae (Coleoptera) de México. Illustrated guide to the genera of Staphylinidae (Coleoptera) of México. Universidad de Guadalajara y CONABIO, México. 401 pp.

Pedraza, M. del C., Márquez, J., Gómez-Anaya, J.A. 2010. Estructura y composición de los ensamblajes estacionales de coleópteros (Insecta: Coleoptera) del bosque mesófilo de montaña en Tlanchinol, Hidalgo, México, recolectados con trampas de intercepción de vuelo. Revista Mexicana de Biodiversidad 81:437-456.

Pfeiler, E., Johnson, s., Richmond, M.P., Markow, T.A. 2013. Population genetics and phylogenetic relationships of beetles (Coleoptera: Histeridae and Staphylinidae) from the Sonoran Desert associated with rotting columnar cacti. Molecular Phylogenetics and Evolution 69:491-501.

Ramírez Reséndiz, F. 2009. Coleópteros necrófilos de las familias Scarabaeidae, Silphidae y Staphylinidae (Insecta: Coleoptera) de los municipios de Huayacocotla e Ilamatlán, Veracruz. Tesis profesional, Licenciatura en Biología, UAEH. Mineral de la Reforma, Hidalgo. 109 pp.

Rodríguez, W.D., Navarrete-Heredia, J.L. 2016. A new Mexican species of Belonuchus Nordmann (Coleoptera, Staphylinidae). Zootaxa 4083(3):444-450.

Ruíz-LizÁrraga, G. 1993. Contribución al conocimiento de los Staphylinidae (Coleoptera) necrófilos de Acahuizotla, Guerrero. Tesis profesional, Licenciatura en Biología, Facultad de Ciencias, UNAM. México, D.F. 277 pp.

Santiago JimÉnez, Q.J. 1999. Los Staphylinidae (Insecta: Coleoptera) necrófilos y coprófilos de un gradiente altitudinal en la región central del estado de Veracruz, México. Tesis profesional, Licenciatura en Biología, Facultad de Biología, Universidad Veracruzana. Xalapa de Enríquez, $126 \mathrm{pp}$.

Sharp, D. 1885. Fam. Staphylinidae. En: Godman, F.D., Salvin, O. (Eds.) Biologia Centrali-Americana, Insecta, Coleoptera. Vol. 1 (2): 393-536. Taylor \& Francis, London.

Sharp, D. 1887. Fam. Staphylinidae. En: Godman, F.D., Salvin, O. (Eds.) Biologia Centrali-Americana, Insecta, Coleoptera. Vol. 1 (2): 673-824. Taylor \& Francis, London.

Smetana, A. 1995. Rove beetles of the subtribe Philonthina of America North of Mexico (Coleoptera: Staphylinidae) classification, phylogeny and taxonomic revision. Memoirs on Entomology, International 3:1-456.

Recibido: 27.03 .2018

Aceptado: 17.05.2018 